\title{
Geodesics and the best measurement for distinguishing quantum states
}

\author{
Åsa Ericsson* \\ Fysikum, Stockholms Universitet, S-106 91 Stockholm, Sweden
}

(Dated: November 26, 2018)

\begin{abstract}
From statistical distinguishability of probability distributions, one can define distinguishability of quantum states. A corresponding measurement to perform, optimal in a definite sense, for distinguishing between two given states $\rho_{A}$ and $\rho_{B}$, has been derived by Fuchs and Caves. We show that the Bures-Uhlmann geodesic through the two states singles out this measurement. The geodesic 'bounces' at the boundary of the set of quantum states. Whenever the geodesic hits the boundary, the state orthogonal to that boundary state is one of the basis states for the measurement.
\end{abstract}

Encountering two quantum states one might ask how similar they are. Clearly one can think of ways to understand 'similar' that would make this question relevant in quantum information processing. One reformulation of the question could be: How well can we distinguish between the two states, with the aid of a measurement? Orthogonal states are one-shot distinguishable - it is possible to measure just once to know the state. But for given non-orthogonal states there is no measurement that will discriminate between the states with certainty. Then we can ask for the measurement that will be the most advantageous one, in a statistical sense, for distinguishing them. For every measurement, the two states give two probability distributions for the outcomes. To distinguish between the states we need to distinguish between the probability distributions.

This leads to statistical distance [1, 2] between states and, in a definite sense, a best distinguishing measurement. Fuchs and Caves [3] have derived an expression for this distance, and also for the operator corresponding to the optimal measurement. The distance turned out to be the same as the geodesic distance of the Bures-Uhlmann metric [4, 5, 6, 7] on the set of quantum states. In this letter we first introduce these results and some facts about the Bures-Uhlmann metric. This will lead the way to our result. We will show how the optimal measurement is determined by the Bures-Uhlmann geodesic connecting the two quantum states: the geodesic 'bounces' at the boundary of the set of quantum states, at states orthogonal to the basis states of the measurement operator.

Every measurement that can be performed on a quantum system can be described by a POVM - a positive operator valued measure. This is a complete set of non-negative Hermitian operators $E_{i}$ acting on an $N$ dimensional Hilbert space $\mathcal{H}_{N} ; i$ indexes the measurement outcomes and completeness means $\sum_{i} E_{i}=\mathbb{1}$ (the identity operator). If the POVM-elements are orthogonal one-dimensional projectors, $E_{i}=\left|e_{i}\right\rangle\left\langle e_{i}\right|$, we have a von Neumann measurement, with a corresponding observable $O=\sum_{i=1}^{N} \lambda_{i} E_{i}$. Upon measuring, the outcome $i$ will occur with probability $p_{i}=\operatorname{Tr} E_{i} \rho$, where $\rho$ is the density operator describing the state of the system. Thus, two states $\rho_{A}$ and $\rho_{B}$ will produce two probability distributions $p^{(A)}$ and $p^{(B)}$.
Sampling from these distributions a finite number of times will give frequencies that differ somewhat from the probabilities. From the size of the statistical fluctuations, a measure of distinguishability between probability distributions can be found. This is the statistical distance $d\left(p^{(A)}, p^{(B)}\right)$, by Bhattacharyya [1] and Wootters [2], given by

$$
\cos d\left(p^{(A)}, p^{(B)}\right)=\sum_{i} \sqrt{p_{i}^{(A)} p_{i}^{(B)}} .
$$

The corresponding Riemannian metric is known as the Fisher-Rao metric.

Different POVMs give different probability distributions, and thus different statistical distance. Take the measurement that gives the maximal statistical distance. Then, by definition, that distance is the distance $d\left(\rho_{A}, \rho_{B}\right)$ between quantum states:

$$
d\left(\rho_{A}, \rho_{B}\right) \equiv \max _{\left\{E_{i}\right\}} \arccos \left(\sum_{i} \sqrt{\operatorname{Tr} E_{i} \rho_{A}} \sqrt{\operatorname{Tr} E_{i} \rho_{B}}\right) .
$$

Fuchs and Caves [3] showed that the maximization yields

$$
d\left(\rho_{A}, \rho_{B}\right)=\arccos \operatorname{Tr} \sqrt{\rho_{A}^{1 / 2} \rho_{B} \rho_{A}^{1 / 2}},
$$

where $\sqrt{\rho}=\rho^{1 / 2}$ is the unique non-negative operator such that $\rho^{1 / 2} \rho^{1 / 2}=\rho$. This expression looks asymmetric, but one can show that it is not. The most separated states are orthogonal states, which rest at the distance $\pi / 2$ from each other. For pure states $\left|\psi_{A}\right\rangle$ and $\left|\psi_{B}\right\rangle$ we have $d=\arccos \left|\left\langle\psi_{A} \mid \psi_{B}\right\rangle\right|$, the angle in Hilbert space between the two vectors.

It may be noted that the same trace expression appears in the fidelity [8]:

$$
F\left(\rho_{A}, \rho_{B}\right)=\left(\operatorname{Tr} \sqrt{\rho_{A}^{1 / 2} \rho_{B} \rho_{A}^{1 / 2}}\right)^{2} .
$$

It is a choice for a quantity that should measure the accuracy of transmission of a signal via a quantum channel.

What is more of our concern here is that the distance $d\left(\rho_{A}, \rho_{B}\right)$ (3) is the same as the geodesic distance in the set of density operators, according to the Bures-Uhlmann 
metric. Shortly we will see how this metric is constructed. But first we will take a look at the best measurementthe measurement found to correspond to the maximal distance (3) 3]. It is a measurement of the observable

$$
M=\rho_{A}^{-1 / 2} \sqrt{\rho_{A}^{1 / 2} \rho_{B} \rho_{A}^{1 / 2}} \rho_{A}^{-1 / 2} .
$$

This optimal measurement is unique, except for some special cases. (These are when $M$ has degenerate eigenvalues, or when the states are non-invertible, i.e. when they are boundary states.) Although we have an explicit expression for the best distinguishing measurement, it is not easy to determine what it is for given density operators. Operator square roots are rather unwieldy to compute. However, this operator has appeared earlier. It is a part of the prescription for Bures-Uhlmann geodesics. We will take advantage of this connection to get a new description of the best measurement.

It should also be mentioned that formula (5) is an operator mean. Operator means, fulfilling a set of reasonable criteria, can be defined for two positive operators $A$ and $B[9]$. The geometric mean,

$$
A \# B=A^{1 / 2} \sqrt{A^{-1 / 2} B A^{-1 / 2}} A^{1 / 2},
$$

is one of these. Thus, we see that the optimal observable (5) is the geometric mean of $\rho_{A}^{-1}$ and $\rho_{B}$ :

$$
M=\rho_{A}^{-1} \# \rho_{B} .
$$

Now we will turn to the Bures-Uhlmann metric [4, 5, 6, 7]. It is obtained from a (kind of) fibre bundle construction in the Hilbert-Schmidt space. From this space of operators $W$, acting on $\mathcal{H}_{N}$, we have the projection

$$
W \longrightarrow \rho=W W^{\dagger},
$$

to the base manifold of positive operators. ( $W^{\dagger}$ denotes the Hermitian conjugate of $W$.) The fibres are obtained by right multiplication of the unitary group, since $W$ and $W U$, for unitary $U$, will be projected to the same operator. In the bundle space we define distances $D\left(W_{A}, W_{B}\right)$ by

$$
D^{2}\left(W_{A}, W_{B}\right)=\operatorname{Tr}\left(W_{A}-W_{B}\right)\left(W_{A}^{\dagger}-W_{B}^{\dagger}\right)
$$

-a Euclidean distance. In the base manifold of positive operators, the distance between two operators is defined as the length of the shortest path between the corresponding fibres in the bundle space. Here we are only interested in the set of normalized density operators, which means projections of operators $W$ on the unit sphere, $\operatorname{Tr} W W^{\dagger}=1$, in the Hilbert-Schmidt space. With this restriction, the geodesic distance we get in the set of density operators is given by equation (3).

The physical interpretation of this construction is that of state-purification. Every mixed state $\rho$ can be purified in a larger Hilbert space; the system is regarded as s subsystem of a bipartite system. The Hilbert-Schmidt space takes the role of the larger state space. Every operator $W$ represents a pure state vector, and the reduced density operator for the subsystem is $W W^{\dagger}$. $W$ is said to be a purification of $\rho$, if $\rho=W W^{\dagger}$. Consequently the whole fibre $W U$ consists of purifications of $\rho$. The distance between the states $\rho_{A}$ and $\rho_{B}$ of the subsystem should not be larger than between any two purifications $W_{A}$ and $W_{B}$. This is assured by finding the shortest path between the fibres of purifications []].

Geodesics on the unit sphere (i.e. great circles) in the Hilbert-Schmidt space can be expressed in the following way:

$$
W(t)=W_{0} \cos t+\dot{W}_{0} \sin t, \quad 0 \leq t<2 \pi,
$$

with normalization and orthogonality conditions, $\operatorname{Tr} W_{0} W_{0}^{\dagger}=\operatorname{Tr} \dot{W}_{0} \dot{W}_{0}^{\dagger}=1$ and $\operatorname{Tr}\left(W_{0} \dot{W}_{0}^{\dagger}+W_{0}^{\dagger} \dot{W}_{0}\right)=0$. For this curve to project to a geodesic in the set of density operators, it is also required that it is everywhere perpendicular to the fibres - the 'horizontality condition'. This reads

$$
\dot{W}_{0}^{\dagger} W_{0}=W_{0}^{\dagger} \dot{W}_{0} .
$$

The geodesic of density operators is then the curve

$$
\rho(t)=W(t) W^{\dagger}(t), \quad 0 \leq t<\pi .
$$

The projected curve will do two turns, since $W$ and $-W$-always resting on the same geodesic - will be projected to the same density operator. Hence, the range for $t$ is halved.

Let us consider the geodesic between the states $\rho_{A}$ and $\rho_{B}$. Assume $W_{A}$ to be a preimage of $\rho_{A}$. Using the 'horizontality condition' (11) (and positivity of $\rho_{A}$ ), it can be shown that the operator $W_{B}$, that is, the preimage of $\rho_{B}$, should be given by

$$
W_{B}=M W_{A},
$$

where $M$ is the positive operator given by (5) - the operator corresponding to the best distinguishing measurement.

Now we start the geodesic at $\rho_{A}$ (assumed to be invertible) and let it go through $\rho_{B}$, resting at the geodesic distance $d$ away. This means that we set

$$
\left\{\begin{array}{l}
W_{A}=W(0), \\
W_{B}=W(d), \text { where } \cos d=\operatorname{Tr} \sqrt{\rho_{A}^{1 / 2} \rho_{B} \rho_{A}^{1 / 2}} .
\end{array}\right.
$$

Inserting in (10), we can solve for the geodesic $W(t)$ in terms of $W_{A}$ and $W_{B}$ :

$$
W(t)=W_{A} \cos t+\left(W_{B}-W_{A} \cos d\right) \frac{\sin t}{\sin d} .
$$

Alternatively, if we use $W_{B}=M W_{A}$, we can express $W(t)$ in terms of $W_{A}$ and $M$ :

$$
W(t)=\left(\mathbb{1} \cos t+(M-\mathbb{1} \cos d) \frac{\sin t}{\sin d}\right) W_{A} .
$$


Here $d$ should be understood as given by $\cos d=\operatorname{Tr} M \rho_{A}$. Finally we have, for the projected curve [10],

$$
\begin{gathered}
\rho(t)=X(t) \rho_{A} X(t), \\
\text { where } X(t)=\left(\mathbb{1} \cos t+(M-\mathbb{1} \cos d) \frac{\sin t}{\sin d}\right) .
\end{gathered}
$$

In this form we can think of a geodesic as given by a starting point $\rho_{A}$ and a positive matrix $M$, determining the direction from $\rho_{A}$.

This shows that there is a close relation between the geodesic and the operator $M$ for the optimal distinguishing measurement. It is then natural to ask: To what extent does the geodesic determine the best measurement? First we note that the measurement is given by the eigenbasis of $M$, while the eigenvalues are superfluous information. To answer the question we need to know more about the geodesics. In one of Uhlmann's papers [7] it is explained how the Bures-Uhlmann geodesics 'bounces' at the boundary of the set of density operators. We will now investigate this feature with the purpose of proving a geometric description of the optimal measurement.

At the boundary of the set of quantum states the matrices $\rho$ have at least one zero eigenvalue, hence $\operatorname{det} \rho=0$. Consider the states on a geodesic, given by equation (17).

$$
\begin{gathered}
\operatorname{det} \rho(t)=0 \quad \Leftrightarrow \quad \operatorname{det} X(t)=0 \\
\Leftrightarrow \quad \operatorname{det}(M-\mathbb{1} x)=0 \\
\text { where } \quad x=\cos d-\cot t \sin d
\end{gathered}
$$

The solutions for $x$ of this equation are the eigenvalues $\lambda_{i}$ of $M$. For the corresponding values of $t$, the states $\rho(t)$ lie on the boundary. These states are

$$
\rho\left(t_{i}\right)=\left(M-\mathbb{1} \lambda_{i}\right) \rho_{A}\left(M-\mathbb{1} \lambda_{i}\right) \frac{\sin ^{2} t_{i}}{\sin ^{2} d} .
$$

( $t_{i}$ is given by the equation $\lambda_{i}=\cos d-\cot t_{i} \sin d$.) There are $N$ or less boundary states, since it is the same as the number of different eigenvalues of $M$. And the number of zero eigenvalues of $\rho\left(t_{i}\right)$ is the same as the degeneracy of the relevant eigenvalue of $M$. When the projected curve reaches the boundary of the set of density matrices it will bounce back into the interior. After $N$, or sometimes less, bounces, the curve will return to its starting point. Thus, the geodesic will consist of a number of segments with endpoints at the boundary of the set of density matrices.

From the expression (19), it is easily recognized what states are orthogonal to geodesics boundary states $\rho\left(t_{i}\right)$ - they are nothing but the eigenvectors $\left|m_{i}\right\rangle$ of $M$ :

$$
\left\langle m_{i}\left|\rho\left(t_{i}\right)\right| m_{i}\right\rangle=0 \text {. }
$$

If all eigenvalues $\lambda_{i}$ are distinct, we get $N$ boundary states, each with one zero eigenvalue, which singles out the $N$ basis states $\left|m_{i}\right\rangle$. If there are degeneracies we get less boundary states, but the sum of the zero eigenvalues is still $N$. And a state $\rho\left(t_{i}\right)$ with $n$ zero eigenvalues is orthogonal to an $n$-dimensional subspace of the pure states. In this subspace any basis can be chosen; $M$ is diagonal in anyone of them. Thus, the geodesic singles out the basis states - the optimal measurement is fully determined by the geodesic.

For two-level systems this provides a practical method for finding the optimal measurement. Every pair of states then lies in a disc in the Bloch ball, which is isometric to a round hemisphere [1]. The geodesics on the hemisphere are just great circles and the relation to the disk is by orthographic projection. In this case the geodesic's endpoints at the 'equator' are the basis states of the measurement. For higher dimensional Hilbert spaces, the picture is much more complex.

In conclusion we have seen how the Bures-Uhlmann geodesics in the set of quantum states bounces at the boundary in a set of $N$, or less, states. If there are $N$ boundary states, the states orthogonal to these form an orthonormal basis in the Hilbert space. If there are less than $N$ boundary states there are just enough lower rank states, so that it is again possible to form an orthonormal basis of states orthogonal to the boundary states. We have shown that such a basis is the basis of an optimal distinguishing measurement: the best measurement-in a specific statistical sense - to perform for distinguishing between two quantum states lying on a segment of the geodesic. This result is a conceptually interesting characterization of the optimal distinguishing measurement.

I gratefully acknowledge many discussions with Ingemar Bengtsson. He has taught me about the BuresUhlmann geometry and encouraged me to do this work.

Note added in proof: An expression for the geodesics, similar to equation (17), has also been derived by Barnum [12].

* asae@physto.se

[1] A. Bhattacharyya, Bull. Calcutta Math. Soc. 35, 99 (1943).

[2] W. K. Wootters, Phys. Rev. D 23, 357 (1981).

[3] C. A. Fuchs and C. M. Caves, Open Sys. \& Information Dyn. 3, 345 (1995).

[4] D. J. C. Bures, Trans. Am. Math. Soc. 135, 199 (1969).

[5] A. Uhlmann, Rep. Math. Phys. 9, 273 (1976).

[6] A. Uhlmann, Rep. Math. Phys. 24, 229 (1986).

[7] A. Uhlmann, Rep. Math. Phys. 33, 253 (1993).

[8] R. Jozsa, J. Mod. Optics 41, 2315 (1994).

[9] T. Ando, Lin. Alg. Appl. 199, 17 (1994).

[10] Hans-Jürgen Sommers, private communication.

[11] M. Hübner, Phys. Lett. A 163, 239 (1992).

[12] H. N. Barnum, PhD Thesis, University of New Maexico (1998). 